\title{
Effective Hemostasis During Minor Surgery in a Case of Hereditary Combined Deficiency of Vitamin K-dependent Clotting Factors
}

Mario Lapecorella, MD, Mariasanta Napolitano, MD, Francesco Bernardi, MS, Mirko Pinotti, PhD, Piero Sandro Sbrighi, MD, Giovanna Marchetti, MS, Alessandro Canella, MS, Pierpaolo Caruso, MS, Assunta Orecchioni, MD, and Guglielmo Mariani, MD

Combined deficiency of the vitamin K-dependent clotting factors (VKCFD) is a rare bleeding disorder involving defective gamma-carboxylation of coagulation factors II , VII, IX and X as well as natural anticoagulants protein $\mathrm{C}$ and protein $\mathrm{S}$. The disease is characterized by a cluster of different, often life threatening, bleeding symptoms occurring both spontaneously and in a surgical setting. In the present paper we describe two different treatment modalities to be used both in a programmed surgical procedure and in an emergency scenario. As this disease is a natural model that resembles oral anticoagulation, our experience discloses a possible rationale in the use of recombinant activated FVII for warfarin reversal.

Keywords: VKFCD; VKORC1 deficiency; surgery; oral anticoagulation $\longrightarrow$ ombined deficiency of the vitamin $\mathrm{K}$-dependent clotting factors (VKCFD) is a rare bleeding disorder involving defective gamma-carboxylation of coagulation factors II (FII), VII (FVII), IX (FIX) and X (FX) as well as natural anticoagulants Protein C (PC) and Protein S (PS) ${ }^{1}$. Vitamin K-dependent clotting factors (VKCFD) is inherited as an autosomal recessive trait and is due to mutations in the g-glutamil carboxylase ${ }^{2}$ (vitamin K-dependent clotting factor type 1) or a defect in the vitamin $\mathrm{K} \mathrm{2,3}$ epoxide reductase complex (vitamin K-dependent clotting factor type 2), which limits the

From the Haemophilia and Thrombosis Centre, University of L'Aquila, Italy (ML, MN, AO, GM); Department of Biochemistry and Molecular Biology, University of Ferrara, Italy (FB, MP, GM, AC, PC); Transfusion Service, Colleferro Hospital, Colleferro, Italy (PSS).

Address correspondence to: Mario Lapecorella, Centro Emofilia e Trombosi, Unità Operativa Medicina Interna 2, Ematologia, Università de L'Aquila, Ospedale San Salvatore, Via Vetoio 1, 67100 Coppito, L’Aquila, Italy; e-mail: emofilia.aq@cc.univaq.it. concentration of the vitamin $\mathrm{K}$ hydroquinone, an essential cofactor of the g-glutamil carboxylase. In vitamin K-dependent clotting factor type 2 , only one causative missense change has been reported to date and particularly a single mutation (Arg98Trp) in the vitamin $\mathrm{K} 2,3$ epoxide reductase complex subunit 1 because of a c. $292 \mathrm{C}>\mathrm{T}$ transition in the VKORC 1 gene. $^{3}$

Vitamin K-dependent clotting factor type 1 (VKCFD) is an extremely rare disease with less than 20 kindreds reported so far. Consequently, very few data exist about the management of the disease, which is characterized by a cluster of different, often life-threatening, bleeding symptoms occurring both spontaneously and in a surgical setting. ${ }^{4}$ In the current article, we report the case of a young woman affected by VKORC1 homozygous deficiency and simultaneously homozygous carrier for the -323 ins 10 mutation in factor VII (FVII) gene, undergoing minor surgical procedures. We display 2 different treatment modalities to be used both in a 


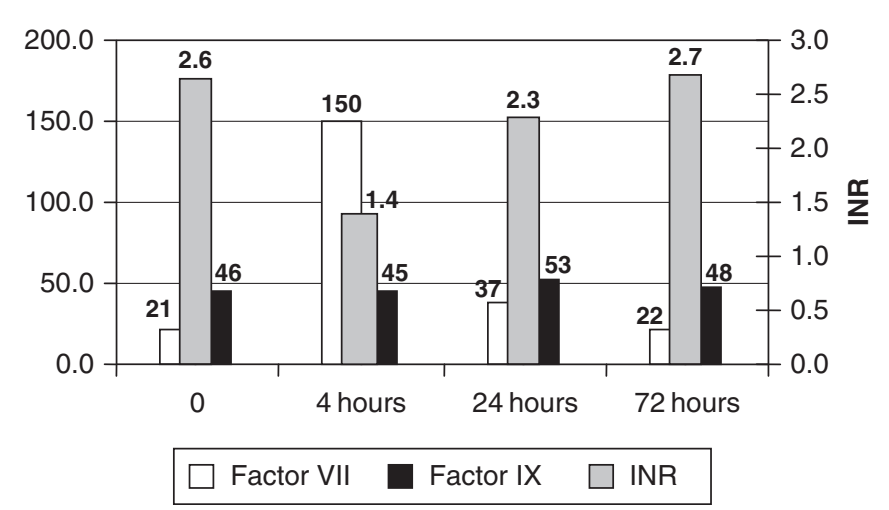

Figure 1. Factor VII and FIX plasma levels and PT-INR values after rFVIIa infusion (1.2 mg total dose). FIX indicates factor FIX; PT-INR, prothrombin time, international normalized ratio; rFVIIa, recombinant activated FVII.

programmed surgical procedure and in an emergency scenario.

\section{Case Report}

A 35-year-old woman requiring dental extractions was admitted to our haemophilia centre from the emergency room of our hospital because of prolongation of the prothrombin time, international normalized ratio (PT-INR; 2.63). Activated partial thromboplastin time (aPTT) was also slightly prolonged (37 seconds). She had a history of frequent nose and gum bleeds, menorrhagia, a life-threatening postpartum hemorrhage at the age of 23 and, more recently, of hysterectomy for hemoperitoneum following ovarian cyst rupture. In addition, she presented with osteoporosis, despite the presence of 1 ovary that had not been removed at the time of hysterectomy. She had been diagnosed as FVII-deficient previous to our observation and consequently treated with a plasmaderived FVII concentrate when needed. Her father and both her brother and sister were asymptomatic with normal values of routine coagulation tests and FVII plasma levels. Her mother died in our section of a liver cirrhosis because of alcohol consumption 1 year after our first observation of the patient, in that coagulation tests were altered in accordance with an advanced liver disease. Mother's personal bleeding history was negative.

The patient's coagulation factor assays performed in our laboratory revealed reduced levels of FII (37\%), FVII (21\%), FIX (46\%), and FX (18\%). To prevent bleeding during the intervention of dental extraction,

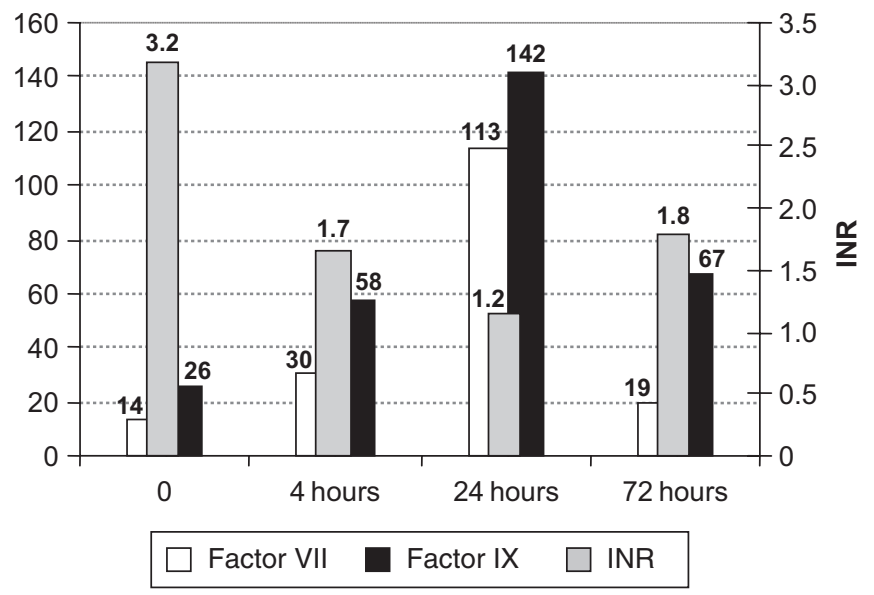

Figure 2. Factor VII and FIX plasma levels and PT-INR values after vitamin $\mathrm{K}$ infusion (10 mg total dose). FIX indicates factor FIX; PT-INR, prothrombin time, international normalized ratio.

30 minutes before starting the procedure, the patient was treated with a single bolus of recombinant activated FVII (rFVIIa; NovoSeven Novonordisk) administrated intravenously at the dose of $20 \mu \mathrm{g} / \mathrm{kg}$ (total dose $1.2 \mathrm{mg}$ ). Coagulation parameters were monitored at $0,4,24$, and 72 hours. As shown in Figure 1, 4 hours after the infusion, an expected increase up to normal levels of FVII activity was reached. An increase of FIX activity and an improvement of PT-INR values (as expected because of the influence of FVII) were obtained as well. The 4-hour assay revealed a double rate increase also of FII and FX (data not shown). The intervention was ruled without any bleeding complication, requiring no further treatment. Clotting factors plasma levels returned to basal levels 24 hours after rFVIIa infusion (Figure 1).

To better define the underlying bleeding disorder, clotting factor assays were repeated after adequate pharmacologic washout displaying similar results. In addition, protein $\mathrm{C}$ (PC) and free protein S (PS) assays were performed revealing low plasma levels. Such findings, along with the patient's bleeding history, were in accordance with a clinical diagnosis of VKCFD. Diagnosis was confirmed with a DNA analysis involving direct scanning of both $\gamma$-glutamil carboxylase and VKORC1 genes and searching for common polymorphisms influencing coagulation factor levels. Results revealed our patient to be homozygous for the c. $292 \mathrm{C}>\mathrm{T}$ transition in the $V K O R C 1^{3}$ gene mutation and homozygous for the -323 ins 10 mutation in FVII gene, which is 
associated with a sensible reduction in FVII plasma levels. ${ }^{5}$

Some months later, the same patient was admitted to our division to perform a digestive endoscopy. Laboratory coagulation assays revealed the following basal levels: PT-INR 3.18, aPTT 33 seconds, FII $16.6 \%$, FVII 14\%, FIX 26\%, FX 7.9\%, PC 36\%, and PS $27 \%$. We challenged the effect of a single vitamin $\mathrm{K} 1$ dose of $10 \mathrm{mg}$ administrated intravenously on clotting factor levels, which were measured at 0,4 , 24, and 72 hours. As shown in Figure 2, a partial response to the vitamin $\mathrm{K}$ intake started at 4 hours and a complete restoration in both FVII and FIX activity was observed at 24 hours with complete normalization of the PT-INR. The 72-hour survey revealed a still shortened PT-INR compared to basal levels. All other factors behaved similarly (data not shown), and gastroscopy with multiple biopsies was performed without bleeding complications the day after vitamin $\mathrm{K}$ administration.

In conclusion, we explored two different treatment modalities of a very rare bleeding disorder, providing further information on the clinical management of VKCFD both in emergency and election settings. To our knowledge, the use of rFVIIa in patients affected by VKCFD has never been reported so far. The elevation of coagulation factors other than FVII remarks the role of rFVIIa as a potent activator of the whole coagulation system, a function that this rare disorder gave us the chance to explore mainly because of the low levels of more than 1 factor. Noticeably, because the two treatments exert their highest effect at different times (4 hours and 24 hours, respectively), it is likely that they can be used consecutively to obtain a sustained normalization of clotting times for major and more complex surgical procedures. Deficiency in VKORC1 has recently captured the interest of the scientific community as mutations other than the Arg98Trp influence the anticoagulant response subsequent to vitamin $\mathrm{K}$ intake and this disease is a natural model that resembles oral anticoagulation. In addition, the usefulness of rFVIIa for warfarin reversal is still debated. ${ }^{6}$ Our experience discloses a possible rationale in the use of this drug which is powerful but whose effects are limited by the short half-life.

\section{Acknowledgments}

Part of the data in Figure 2 was used for an in vitro kinetic study meant to measure variation in vitamin $\mathrm{K}$-dependent clotting factors after a vitamin $\mathrm{K}$ load, which was published in JTH in May 2008. The authors would like to thank the Fondazione Cassa di Risparmio di Ferrara (CARIFE) for the financial support.

\section{References}

1. Hansson K, Stenflo J. Post-translational modifications in proteins involved in blood coagulation. J Thromb Haemost. 2005;3:2633-2648.

2. Zhang B, Ginsburg D. Familial multiple coagulation factor deficiencies: new biologic insight from rare genetic bleeding disorders. J Thromb Haemost. 2004;2: 1564-1572.

3. Rost S, Fregin A, Ivaskevicius V, et al. Mutations in VKORC1 cause warfarin resistance and multiple coagulation factor deficiency type 2. Nature. 2004;427:537-541.

4. Bhattacharyya J, Dutta P, Mishra P, et al. Congenital vitamin K-dependent coagulation factor deficiency: a case report. Blood Coagul Fibrinolysis. 2005;16:525-527.

5. Bernardi F, Marchetti G, Pinotti M, et al. Factor VII gene polymorphisms contribute about one third of the factor VII level variation in plasma. Arterioscler Thromb Vasc Biol. 1996;16:72-76.

6. Hanley JP. Warfarin reversal. J Clin Pathol. 2004;57: 1132-1139. 